\title{
Lactoferrin for the Prevention of Post-antibiotic Diarrhoea
}

\author{
Alison M. Laffan', Robin McKenzie², Jennifer Forti³, Dawn Conklin³, Richard Marcinko3, \\ Ruchee Shrestha ${ }^{3}$, Michele Bellantoni ${ }^{3}$, and William B. Greenough III ${ }^{3}$ \\ 'Department of Epidemiology, ${ }^{2}$ Division of Infectious Diseases, and ${ }^{3}$ Division of Geriatric Medicine, \\ Johns Hopkins University, Baltimore, MD 21224, USA
}

\begin{abstract}
Antibiotic-associated diarrhoea (AAD) is a common cause of morbidity and mortality. Older individuals in long-term care facilities are particularly vulnerable due to multisystem illnesses and the prevailing conditions for nosocomial infections. Lactoferrin, an antimicrobial protein in human breastmilk, was tested to determine whether it would prevent or reduce $\mathrm{AAD}$, including Clostridium difficile in tube-fed long-term care patients. Thirty patients were enrolled in a randomized double-blind study, testing eight weeks of human recombinant lactoferrin compared to placebo for the prevention of antibiotic-associated diarrhoea in long-term care patients. Fewer patients in the lactoferrin group experienced diarrhoea compared to controls $(\mathrm{p}=0.023)$. Based on the findings, it is concluded that human lactoferrin may reduce post-antibiotic diarrhoea.
\end{abstract}

Key words: Antibiotics; Clostridium difficile; Colitis; Diarrhoea; Diarrhoea, Drug-induced; Elderly; Lactoferrin

\section{INTRODUCTION}

Up to $25 \%$ of patients treated with broad-spectrum antibiotics experience diarrhoea (1), and this antibiotic-associated diarrhoea (AAD) can vary from mild nuisance loose or watery stools to severe diarrhoea, including colitis. Patients in long-term care are particularly vulnerable, and AAD is an important cause of disability and death in this population (2). However, Clostridium difficile, a known aetiologic agent, explains less than half of these cases. The consequences of AAD can be severe, leading to debility due to volume depletion, enteral protein loss, and a chronic inflammatory state (3). Ironically, $\mathrm{AAD}$ is also being treated with antibiotics and may contribute to the growing problem of antibiotic-resistant pathogens (4). To prevent postantibiotic diarrhoea, we employed a normal human breastmilk protein-lactoferrin-which has both anti-inflammatory and antimicrobial properties (5) and has recently been shown to reduce

Correspondence and reprint requests should be addressed to:

Prof. William B. Greenough III

5505 Hopkins Bayview Circle

Baltimore, MD 21224

USA

Email:wgreeno2@jhmi.edu

Fax: 410-550-2513

Alternate corresponding author:

Dr. Alison M. Laffan

Email: alaffan14@gmail.com the duration of diarrhoea in children (6). We tested whether recombinant human lactoferrin grown in rice could prevent post-antibiotic diarrhoea in long-term care residents.

\section{MATERIALS AND METHODS}

Nursing-home residents were randomized to receive, by gastrostomy tube, lactoferrin $(5 \mathrm{mg} / \mathrm{mL})$ or placebo via a flush solution. Both product and placebo were dissolved in $600 \mathrm{~mL}$ of $0.3 \%$ saline solution and were similar in appearance, ensuring that patients and staff be blind to treatment condition. The solution was administered each day for 56 days. Nurses and nursing assistants recorded stool quality on each shift, and stool samples were tested for $C$. difficile at enrollment, day 14, 42, and 56.

We conducted an interim analysis when $50 \%$ of the enrollment goal was completed (16 participants), leading to a change in the inclusion criteria and the randomization scheme. Thus, the study was divided into two phases-phase 1 and phase 2. During phase 1 , patients initially colonized with C. difficile (C. difficile antigen present in stool samples) were excluded from participation, leading to $38 \%(6 / 16)$ of the participants being disenrolled immediately. Phase 2 amended the protocol such that the participants be only excluded if they had clinically-confirmed disease due to $C$. difficile 
(stool samples testing positive for $C$. difficile antigen and toxin A and B). Since all participants who were excluded from the study due to $C$. difficile colonization were from the lactoferrin group (6/6), we altered the randomization scheme in phase 2 . Phase 2 randomization was changed to two lactoferrin enrollees for each placebo enrollee to ensure a large sample-size enough for comparison between lactoferrin and control groups.

\section{Study subjects}

Patients at the 41-bed ventilator rehabilitation unit of the Johns Hopkins Geriatric Center beginning a new course of broad-spectrum antibiotics (not including metronidazole, vancomycin, and linezolid) were approached for participation in this study, if they had met the following inclusion criteria: (a) nutrition via an enteral feeding-tube; (b) free from antibiotics for 10 days before entry into the study; (c) not allergic to rice or rice products; and (d) not colonized with C. difficile (phase 1) or no signs or symptoms of clinically-confirmed $C$. difficile-assoc-iated disease (phase 2).

\section{C. difficle testing}

All stool samples were tested for $C$. difficile antigens using a rapid enzyme immunoassay Techlab (CDIFF-CHEK). Samples testing positive for $C$. difficile antigens were further tested for cytotoxins using a cell culture cytotoxicity assay (7). Samples testing positive for $C$. difficile antigen and toxin $A$ and B were classified as infected with $C$. difficile. Samples that tested positive for $C$. difficile antigen only were classified as colonization.

\section{Statistical analysis}

Diarrhoea was defined as two or more loose stools (conforming to the shape of a container) within a 24-hour period. Each 24-hour period meeting this criterion was classified as a diarrhoea-day. Comparisons between lactoferrin and control groups were made on the mean and the total number of diarrhoea-days. Further, an episode of diarrhoea was defined as consecutive days of diarrhoea, ending after two successive 24-hour periods without diarrhoea. The participants were also compared on time-to-first episode of diarrhoea, or time from enrollment into the study to the first diarrhoea-day. The participants never experiencing diarrhoea were censored at the last day of participation in the study as diarrhoea-free.

Non-parametric tests were used for comparisons between lactoferrin and control groups because of the small sample-sizes and failure to meet normality assumptions for continuous data. Categorical variables were compared using Fisher's exact test, and statistical significance was assessed using the
Wilcoxon signed-rank test for continuous parameters. The group differences in time-to-diarrhoea were assessed using the log-rank test.

\section{Ethical aspects}

Informed consent was obtained from the patient or healthcare agent before enrollment. The Institutional Review Board of the Johns Hopkins Medicine approved the study.

\section{RESULTS}

Data relating to stool for 22 participants (13 control and 9 lactoferrin participants) were analyzed. The remaining eight participants were excluded from analysis because they did not complete the study. Each of these participants was exited from the study during the first week of participation, and the large majority (6/8) was exited from the study immediately because of a positive $C$. difficile antigen test results, during phase 1 of the study. Two other participants were disenrolled when they were found to have clinical infections due to $C$. difficile. These infections, noted at day 3 and 8 , were classified as existing before enrollment into the study by the principal investigator (WBG).

The table compares the lactoferrin group with the control group at enrollment. No significant differences were found in comparisons between the two groups. However, shorter times since the last antibiotic therapy were observed in the lactoferrin group $(\mathrm{p}=0.07)$, and these participants were more likely to be enrolled in the second phase of the study $(\mathrm{p}=0.07)$.

Fewer patients $(4 / 9 ; 44.4 \%)$ in the lactoferrin group experienced diarrhoea compared to the control group $(12 / 13 ; 92.3 \% ; \mathrm{p}=0.023)$. Those treated with lactoferrin were at a significantly reduced risk of experiencing diarrhoea compared to the participants in the control group [odds ratio $(\mathrm{OR})=0.07,95 \%$ confidence interval (CI) 0.001-0.97]. Comparisons of the mean number of diarrhoea-days (control: 9.3 vs lactoferrin: 4.0 days, $\mathrm{p}=0.072$ ) and the percentage of the study days with diarrhoea (control: $17.1 \%$ vs lactoferrin: $8.0 \%, \mathrm{p}=0.068$ ) showed a trend towards more diarrhoea in the control group. In addition, time-to-diarrhoea was shorter for the control group compared to the lactoferrin group (median time control: 7 vs lactoferrin: $60+$ days, $\mathrm{p}=0.067$ ). This trend can be seen in the figure, which shows the proportion of the participants free from diarrhoea as a function of time since enrollment into the study for lactoferrin (dashed line) and control (solid line) groups.

During the study period, five participants ( 2 control and 3 lactoferrin) became infected with $C$. diffi- 


\begin{tabular}{|c|c|c|c|c|c|}
\hline \multirow[b]{2}{*}{ Characteristics } & \multicolumn{2}{|c|}{ Lactoferrin $(\mathrm{n}=9)$} & \multicolumn{2}{|c|}{ Control $(\mathrm{n}=13)$} & \multirow[b]{2}{*}{$\mathrm{p}$ value } \\
\hline & $\begin{array}{l}\text { Mean } \\
\text { or no. }\end{array}$ & $\begin{array}{c}\text { Range } \\
\text { or \% }\end{array}$ & $\begin{array}{c}\text { Mean or } \\
\text { no. }\end{array}$ & $\begin{array}{c}\text { Range } \\
\text { or } \%\end{array}$ & \\
\hline Age (years) & 62.1 & $23.2-83.9$ & 62.4 & $34.8-91.1$ & 0.92 \\
\hline Male (no., \%) & 5 & 55.6 & 3 & 23.1 & 0.38 \\
\hline Black (no., \%) & 2 & 22.2 & 5 & 38.5 & 0.65 \\
\hline Anaemia (no.,\%)* & 5 & 55.6 & 4 & 30.8 & 0.24 \\
\hline Time (years) on unit & 0.5 & $0.01-1.9$ & 1.6 & $0.01-6.9$ & 0.35 \\
\hline Time (years) on tube-feeding & 0.85 & $0.14-5.17$ & 0.64 & $0.04-2.54$ & 0.57 \\
\hline Time (days) since last antibiotic & 29 & $18-48$ & 48 & $20-99$ & 0.07 \\
\hline Phase 1 of study (no., \%) & 1 & 11.1 & 7 & 53.9 & 0.07 \\
\hline Time (days) in study & 50.4 & $19-61$ & 45.6 & $25-60$ & 0.25 \\
\hline C. difficile antigen+ (no., \%) & 2 & 22.2 & 2 & 15.4 & 1.00 \\
\hline
\end{tabular}

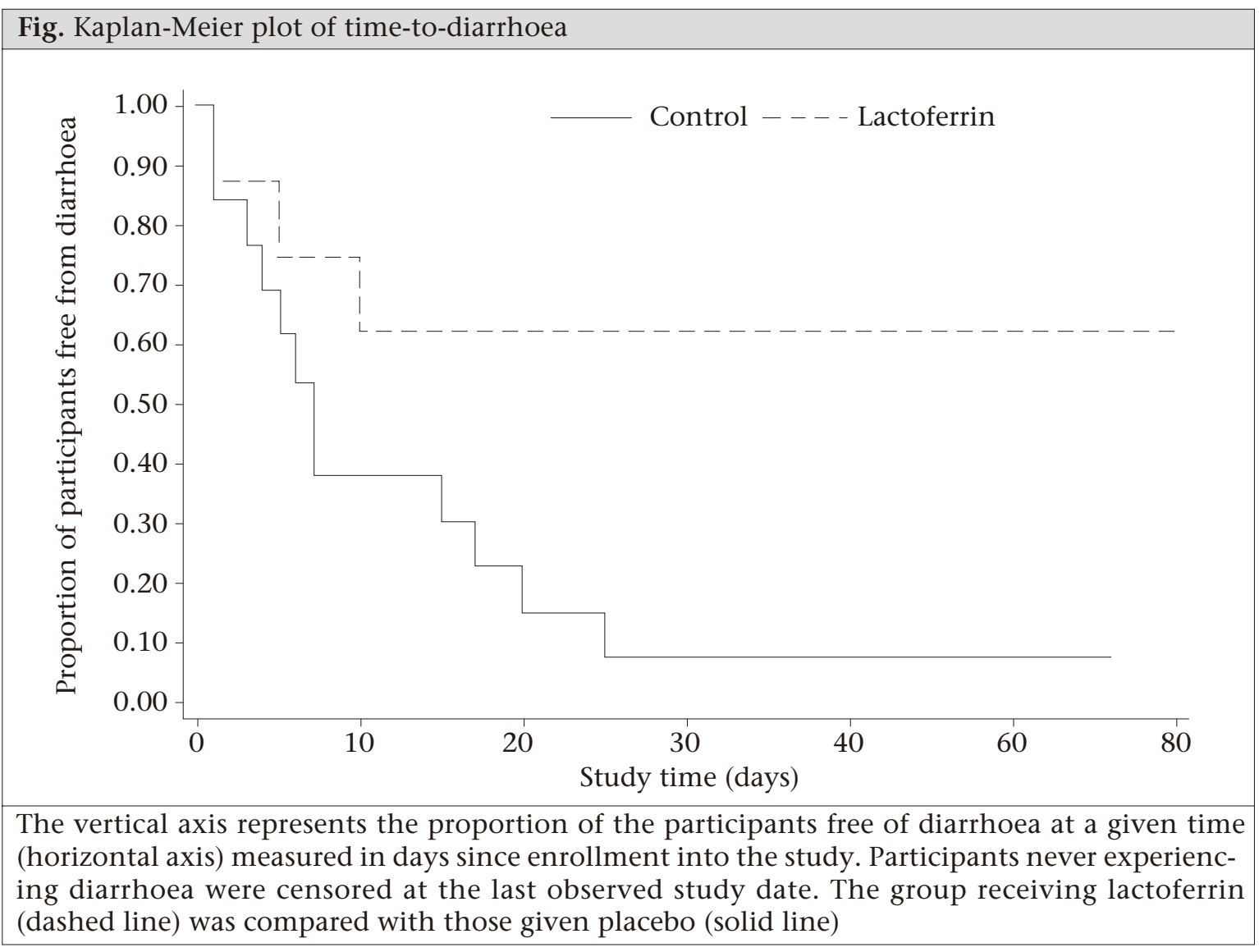

cile (positive for $C$. difficile antigen and toxins), and of these participants, $2 / 2$ in the control group and $1 / 3$ in the lactoferrin group experienced diarrhoea. The differences between control and lactoferrin groups were not significant ( $\mathrm{p}>0.15$ for both).

\section{DISCUSSION}

Antibiotics destroy normal intestinal microbial flo- ra often leading to diarrhoea (8). Older individuals in long-term care settings are particularly vulnerable (9). The risk of morbidity or mortality due to diarrhoeal disease is higher in those aged over 65 years (2). Although $C$. difficile is recognized as an important cause of $\mathrm{AAD}$, evidence of infection due to $C$. difficile is found in less than half of all cases (3), and current treatment requires administration 
of antibiotics. Alternative strategies are needed to prevent or treat $C$. difficile and other AADs. The pilot study reported here tested human recombinant lactoferrin as a preventive tool against post-antibiotic diarrhoea.

Lactoferrin, a normal human protein found in breastmilk and leukocytes, has both antibacterial and anti-inflammatory properties (5). This protein does not damage protective microbial flora of the intestine. Human lactoferrin was prepared by inserting the human gene into rice to prepare large amounts at low cost (10) and is nearly identical to native human lactoferrin (11). Administering high concentrations $(5 \mathrm{mg} / \mathrm{mL}$ ) of this product produced no adverse effects in our population of frail older adults, indicating that lactoferrin is safe.

It was recently reported that recombinant lactoferrin with lysozyme reduced the duration of diarrhoea compared to placebo controls in children when delivered via an oral rehydration solution (6). These results suggest that at least some of the effect of breastfeeding to prevent diarrhoea may be due to lactoferrin (12). Here, we have administered this breastmilk component to older individuals; with a result similar to the benefits to infants. Our results offer an interesting alternative approach to preventing and, perhaps, treating post-antibiotic diarrhoea.

Although not the primary outcome, we did monitor for $C$. difficile-associated infection. There were too few infections due to $C$. difficile to assess the effects of lactoferrin on this important cause of postantibiotic diarrhoea.

In this small study, we had observed a significant reduction in diarrhoea between treatment and placebo groups, suggesting that lactoferrin may be effective in preventing AAD. Caution should be taken in interpreting these results because this study was designed as a pilot project and enrolled a small number of patients. Further, we were forced to alter the study protocol mid-way through the project to achieve balance in treatment groups. However, given our significant findings, this novel preventive approach warrants further investigation in larger trials.

In addition to efficacy trials, more research on the mechanisms through which lactoferrin may work to prevent $\mathrm{AAD}$ is needed. Results of research indicate that human lactoferrin provides a readilyabsorbable source of iron to any patients with iron-deficiency anaemia (13), and this provides a possible additional benefit of lactoferrin administration. We attempted to investigate this hypothesis post-hoc using information on treatment for anaemia (use of ferrous sulphate) and red blood cell counts; however, due to the small sample-sizes and the post-hoc nature of the analysis, we were unable to show an effect of red blood cell counts in these patients. Future studies should consider the inclusion of a full measure of iron status and parameters of anaemia to explicitly test the potential added benefit of administration of recombinant lactoferrin beyond its antibacterial and anti-inflammatory properties.

Lactoferrin when administered at the start of antibiotic treatment reduced the attack rate of diarrhoea over an eight-week period. There were too few instances of $C$. difficile to assess the effects on this cause of post-antibiotic diarrhoea. Our results offer an interesting alternative approach to preventing and, perhaps, treating post-antibiotic diarrhoea. Further research should incorporate larger samplesizes powered to determine any effect on $C$. difficile as a serious complication of antibiotic therapy.

\section{ACKNOWLEDGEMENTS}

Ventria Bioscience, the manufacturer of the lactoferrin flush solution, funded this research project. This funding included a small amount of salary support given to Johns Hopkins University for William B. Greenough III, Robin McKenzie, Richard Marcinko, and Alison M. Laffan. Dr. Delia Bethel, a Ventria scientist advised on protocol design, provided the randomization, and assisted with data entry. All analyses were conducted independent of Ventria Bioscience.

The results of this study were presented at the 2009 meeting of the American Geriatric Society, Chicago, IL, USA.

\section{REFERENCES}

1. Bergogne-Bérézin E. Treatment and prevention of antibiotic associated diarrhea. Int J Antimicrob Agents 2000;16:521-6.

2. Gangarosa RE, Glass RI, Lew JF, Boring JR. Hospitalizations involving gastroenteritis in the United States, 1985: the special burden of the disease among the elderly. Am J Epidemiol 1992;135:281-90.

3. Bartlett JG. Clinical practice. Antibiotic-associated diarrhea. N Engl J Med 2002;346:334-9.

4. Fischbach MA, Walsh CT. Antibiotics for emerging pathogens. Science 2009;325:1089-93.

5. Lönnerdal B, Iyer S. Lactoferrin: molecular structure and biological function. Annu Rev Nutr 1995;15:93110.

6. Zavaleta N, Figueroa D, Rivera J, Sánchez J, Alfaro S, Lönnerdal B. Efficacy of rice-based oral rehydration solution containing recombinant human lactoferrin 
and lysozyme in Peruvian children with acute diarrhea. J Pediatr Gastroenterol Nutr 2007;44:258-64.

7. Ticehurst JR, Aird DZ, Dam LM, Borek AP, Hargrove JT, Carroll KC. Effective detection of toxigenic Clostridium difficile by a two-step algorithm including tests for antigen and cytotoxin. J Clin Microbiol 2006;44:1145-9.

8. Hogenauer C, Hammer HF, Krejs GJ, Reisinger EC. Mechanisms and management of antibiotic-associated diarrhea. Clin Infect Dis 1998;27:702-10.

9. Lew JF, Glass RI, Gangarosa RE, Cohen IP, Bern C, Moe CL. Diarrheal deaths in the United States, 1979 through 1987. A special problem for the elderly. JAMA 1991;265:3280-4.
10. Nandi S, Yalda D, Lu S, Nikolov Z, Misaki R, Fujiyama $\mathrm{K}$ et al. Process development and economic evaluation of recombinant human lactoferrin expressed in rice grain. Transgenic Res 2005;14:237-49.

11. Nandi S, Suzuki YA, Huang JM, Yalda D, Pham P, Wu L et al. Expression of human lactoferrin in transgenic rice grains for the application in infant formula. Plant Sci 2002;163:713-22.

12. Victora CG, Smith PG, Vaughan JP, Nobre LC, Lombardi $\mathrm{C}$, Teixeira AM et al. Infant-feeding and deaths due to diarrhea-a case-control study. Am J Epidemiol 1989;129:1032-41.

13. Lönnerdal B, Bryant A. Absorption of iron from recombinant human lactoferrin in young US women. Am J Clin Nutr 2006:83:305-9. 